NOTA PALEONTOLÓGICA

\title{
Primer registro de microcoprolitos de crustáceos de la Cuenca Neuquina: el icnogénero Palaxius en el Tithoniano de la Formación Vaca Muerta, Mendoza
}

\author{
Diego A. KIETZMANN¹ y Ricardo M. PALMA ${ }^{1}$
}

\section{Introducción}

Algunos grupos de crustáceos producen microcoprolitos estructurados internamente debido a proyecciones de las paredes del intestino (Moore, 1939); el arreglo interno, la forma y el número de canales, vistos en sección transversal, se utilizan como criterio para su clasificación (e.g. Schweigert et al., 1997).

A nivel mundial el registro de microcoprolitos de crustáceos es abundante (e.g. Brönnimann y Norton, 1960; Senowari-Daryan y Kuss, 1992; Schweigert et al., 1997; Senowbari-Daryan y Kube, 2003; Peckmann et al., 2007) y su valor como elementos de correlación estratigráfica ha sido destacado por Blau et al. (1993). En contraposición, la presencia de estos microcoprolitos en América del Sur es escasa y sólo se conocen aquellos provenientes de Perú, Colombia, Venezuela y Chile (Förster y Hillebrandt, 1984; Senowbari-Daryan y Stanley, 1986; Blau et al., 1994; 1995; De Romero y Galea-Álvarez, 1995); la presencia de este tipo de estructuras no ha sido mencionada para la Cuenca Neuquina.

Los depósitos marinos que comprenden el límite Jurásico-Cretácico de la cuenca contienen una rica fauna de invertebrados marinos representados principalmente por moluscos (e.g. Riccardi et al., 2000), en tanto que la fauna de crustáceos y las estructuras que generan es menos conocida. De acuerdo con Aguirre Urreta (1989; 2003) los restos de decápodos provenientes del Grupo Mendoza (figura 1) se encuentran comúnmente preservados como quelípedos aislados e incluyen los géneros Hoploparia, Callianassa, Protocallianassa, Eryma y Meyerella. Entre ellos los callianássidos y protocallianássidos son los más abundantes y fueron reco-

1Universidad de Buenos Aires, Facultad de Ciencias Exactas y Naturales, Departamento de Ciencias Geológicas, Pabellón II. Ciudad Universitaria, 1428 Buenos Aires, Argentina - Consejo Nacional de Investigaciones Científicas y Técnicas (CONICET). diegokietzmann@gl.fcen.uba.ar; palma@gl.fcen.uba.ar

C Asociación Paleontológica Argentina nocidos en el Tithoniano medio de la Formación Vaca Muerta por Leanza y Zeiss (1990), así como en el Berriasiano tardío y Valanginiano temprano (Aguirre-Urreta, 1989).

Estudios estratigráficos detallados en la Formación Vaca Muerta (e.g. Kietzmann et al., 2008; Kietzmann y Palma, 2009) muestran la abundancia de trazas de Thalassinoides Ehrenberg, 1944 en ciertos niveles. Éstas aparecen como sistemas de galerías que poseen una arquitectura de tipo boxwork, comúnmente asociada al género Callianassa (Bromley, 1990).

En este trabajo se presenta el primer registro de microcoprolitos de crustáceos para la Cuenca Neuquina y Argentina, así como el primer registro de Palaxius Brönnimann y Norton, 1960 para el Jurásico Superior y Cretácico Inferior de América del Sur.

\section{Marco geológico, origen y preservación de los ejemplares}

Durante el Jurásico Tardío y Cretácico Temprano, en la Cuenca Neuquina se desarrolló una amplia rampa carbonática de bajo gradiente topográfico cuyas facies distales están representadas por depósitos de cuenca a rampa media y reciben el nombre de Formación Vaca Muerta (Weaver, 1931 enmend. Leanza, 1973; Tithoniano inferior-Valanginiano inferior), mientras que las facies proximales están representadas por depósitos de rampa interna que se conocen como Formación Chachao (Mombrú et al., 1978; Valanginiano inferior) (figura 1). Más información sobre estas unidades puede consultarse en Legarreta y Kozlowski (1981), Legarreta y Uliana (1991), Palma y Lanés (2001), Scasso et al. (2002), Palma et al. (2008) y Kietzmann et al. (2008).

Las muestras que contienen microcoprolitos provienen de distintas secciones estratigráficas de la Formación Vaca Muerta aflorantes en la provincia de Mendoza (figura 1) y han sido obtenidas en niveles del Tithoniano superior y Berriasiano inferior AMGHB2-0002-7014/10\$00.00+.50 


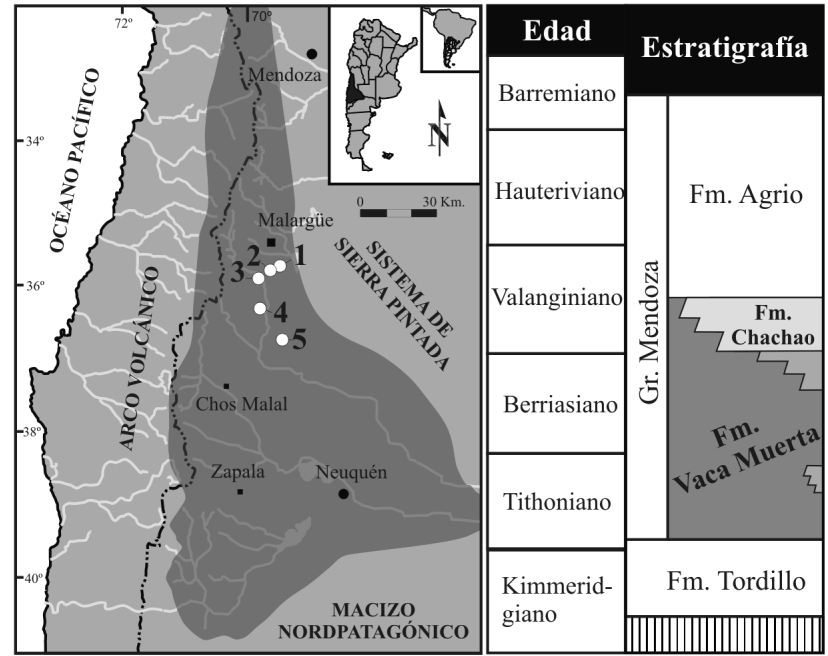

Figura 1. Mapa de ubicación de la Cuenca Neuquina con localidades de estudio y cuadro estratigráfico del Grupo Mendoza. Referencias: 1, Arroyo Loncoche, 2, Cuesta del Chihuido, 3, Bardas Blancas, 4, Sierra Azul, 5, Sierra de la Cara Cura / location map of the Neuquén Basin with study localities and stratigraphic chart of the Mendoza Group. References: 1, Arroyo Loncoche, 2, Cuesta del Chihuido, 3, Bardas Blancas, 4, Sierra Azul, 5, Sierra de la Cara Cura.

(zonas de Corongoceras alternans y Substeueroceras koeneni). Las muestras provienen de packstones y wackestones laminados, ricos en amonites, bivalvos y radiolarios, depositados en facies de rampa externa (e.g. Kietzmann et al., 2008).

La preservación de los microcoprolitos es en general muy pobre. Los canales suelen estar rellenos de fango y los bordes son difusos, probablemente como consecuencia de los efectos de la compactación y el neomorfismo. Por este motivo, los microcoprolitos han pasado desapercibidos y fueron originalmente descriptos como peloides, un componente aloquímico muy común de las micritas peloidales previamente mencionadas por Scasso et al. (2002) y Kietzmann et al. (2008).

El detallado análisis microfacial realizado en los depósitos de la Formación Vaca Muerta permitió reconocer que los mejores ejemplares de microcoprolitos se encuentran rellenando en forma parcial las cámaras de algunos amonites. Dentro de las mismas se advierte un gradiente en la preservación que varía desde el exterior hacia el interior de las cámaras, donde los efectos de la cementación calcítica son mayores. La preservación de los canales es buena cuando las texturas forman grainstones microcoprolíticos (interior de la cámara), mientras que cuando las texturas forman wackestones o packstones (exterior de la cámara), la preservación de los canales internos disminuye considerablemente (figura 2) debido a la mayor disponibilidad de fango.

AMEGHINIANA 47 (2), 2010

\section{Paleontología sistemática}

La condición de los microcoprolitos como unidades independientes en el sistema linneano, así como la clasificación de los crustáceos fósiles está aún en discusión (Blau y Grün, 2000). Aquí se considerará a estos coprolitos como trazas fósiles siguiendo el criterio de Bertling et al. (2006), quienes los consideran estructuras morfológicamente recurrentes que resultan de la modificación del sustrato a partir de la actividad de un organismo individual. Se utilizará además la nomenclatura de Vialov (1978), quien introdujo el término Favreinidae para agrupar y distinguir a los coprolitos de crustáceos de sus productores.

\section{Icnofamilia FAVREINIDAE Vialov, 1978}

Icnogénero Palaxius Brönnimann y Norton, 1960

Icnoespecie tipo. Palaxius habanensis Brönnimann y Norton, 1960.

Diagnosis. El icnogénero Palaxius presenta una morfología cilíndrica y se caracteriza, en sección transversal, por la presencia de canales longitudinales con forma de media luna creciente alrededor de un plano de simetría (Brönnimann y Norton, 1960).

Observaciones. El icnogénero Palaxius contiene 25 icnoespecies conocidas desde el Carbonífero Tardío hasta el Mioceno ( $c f$. Senowbari-Daryan y Kube, 2003; Buchs et al., 2009), de las cuales Palaxius biserialis (Kristan-Tollmann, 1989) ha sido registrada en el Tithoniano, mientras que Palaxius decaochetarius Palik, 1965 y Palaxius tetraochetarius Palik, 1965 en el Berriasiano-Albiano.

\section{Palaxius decaochetarius Palik, 1965}

Figuras 2.3-4

Descripción. Microcoprolito de forma cilíndrica sin surco ventral, con una sección transversal de 1300 a $1500 \mu \mathrm{m}$ y morfología circular a ovalada. Diez canales lo atraviesan en sentido longitudinal. Estos se organizan en dos grupos alrededor de un plano de simetría bilateral (con disposición 2:3-3:2). Cada uno contiene dos canales dorsales (canales 1 y 2) y tres canales ventrales (3, 4 y 5). La forma de los canales es de tipo creciente y cóncava hacia el centro para los canales $1 / 1^{\prime}, 2 / 2^{\prime}$ y $3 / 3^{\prime}$, y creciente cóncava hacia afuera para los canales $4 / 4^{\prime}$ y $5 / 5^{\prime}$ (figura 3 ). La relación angular de los canales respecto del plano de simetría es de $20^{\circ}$ para el canal $1 / 1^{\prime}, 45^{\circ}$ para los canales $2 / 2^{\prime}$ y $4 / 4^{\prime}, 120^{\circ}$ para el canal $3 / 3^{\prime}$ y $90^{\circ}$ para el canal $5 / 5^{\prime}$.

Observación. Se conocen otras cuatro icnoespecies de diez canales del icnogénero Palaxius, de la cuales 

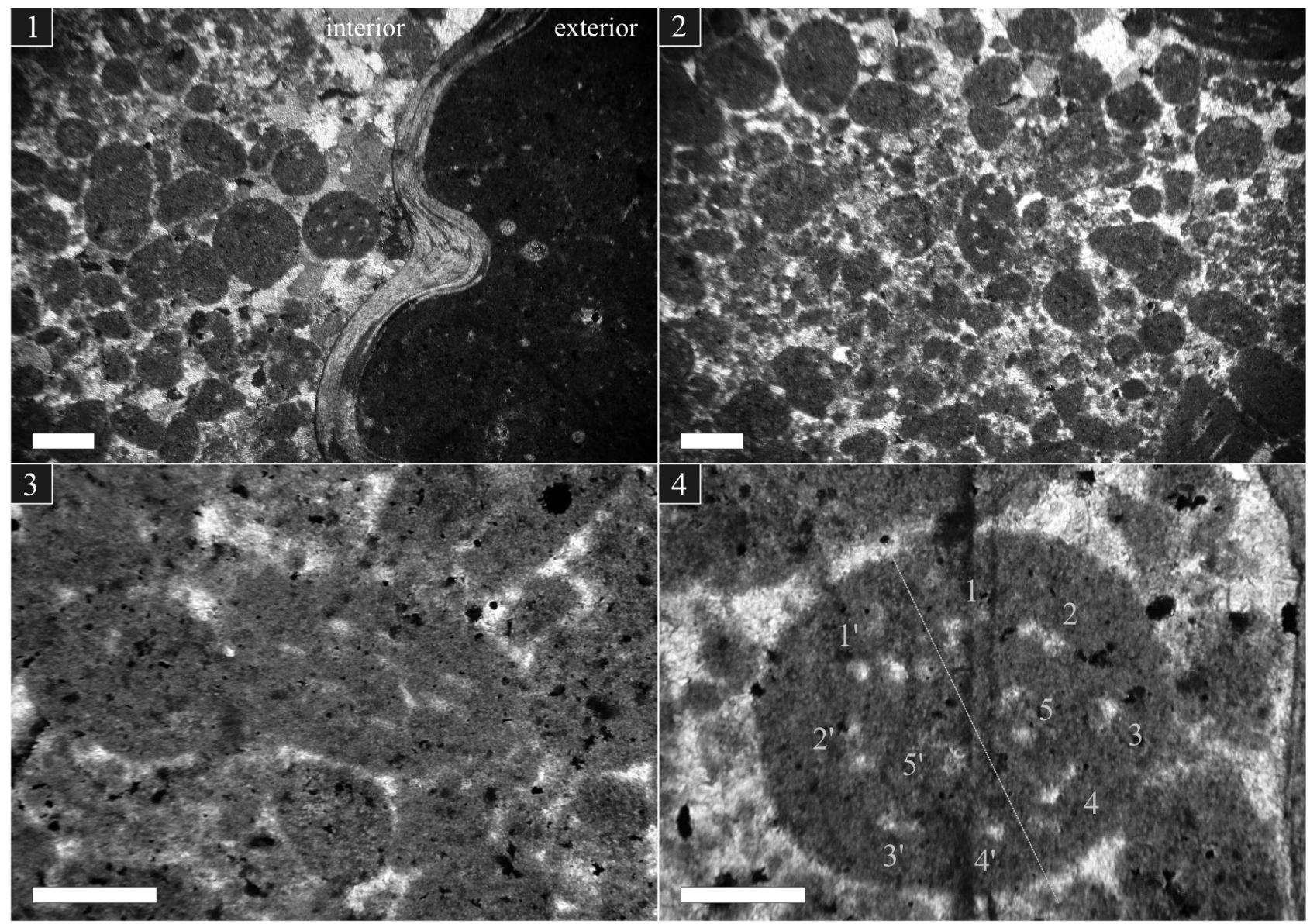

Figura 2. Microcoprolitos del icnogénero Palaxius. 1-2, Microfacies de grainstones/packstones microcoprolíticos. Observar la diferencia de preservación dentro y fuera de la cámara del amonite; 3-4, Secciones transversales de Palaxius decaochetarius Palik. Escala gráfica: 0,5 $\mathrm{mm} /$ microcoprolites of the ichnogenus Palaxius. Note differences in preservation between the inside and outside of the ammonite chamber; 1-2, Microcoprolitic grainstones/packstones microfacies; 3-4, Transversal sections of Palaxius decaochetarius Palik. Scale bar: $0.5 \mathrm{~mm}$.

Palaxius habanensis Brönnimann y Norton, 1960 y $P$. colombiensis Blau, Grün y Senff, 1993 son las que presentan mayor similitud con la aquí descripta. Palaxius decaochetarius se diferencia de Palaxius habanensis por la orientación de los canales $1 / 1^{\prime}$ y $5 / 5^{\prime}$, mientras que $P$. colombiensis por la orientación del canal $1 / 1^{\prime}$ y $4 / 4^{\prime}$ Las diferencias con estas icnoespecies, así como otras icnoespecies de Palaxius pueden consultarse en Blau y Grün (2000).

Distribución estratigráfica y edad. Palaxius decaochetarius se conoce en depósitos del Berriasiano hasta el Albiano de Hungría (Palik, 1965). Los especímenes de la Formación Vaca Muerta provienen de niveles correspondientes a las zonas de Corongoceras alternans (Tithoniano superior bajo) y Substeueroceras koeneni (Tithoniano superior alto-Berriasiano inferior) del extremo sur de la sierra de la Cara Cura. Microcoprolitos similares, pero mal preservados, se reconocieron en niveles equivalentes en el Arroyo Loncoche, Cuesta del Chihuido, Bardas Blancas y Sierra Azul (figura 1).

\section{Discusión}

\section{Productores potenciales}

De acuerdo con Schweigert et al. (1997) los microcoprolitos de crustáceos permiten distinguir productores a nivel de familia, mientras que a nivel genérico o específico la asignación a veces se torna imposible.

Blau et al. (1993) asociaron los icnogéneros Payandea Blau, Grün y Sneff, 1993 y Thoronetia Brönnimann, Caron y Zaninetti, 1972b con la Familia Galatheidae, mientras que los icnogéneros Favreina Brönnimann, 1955, Parafavreina Brönnimann, Caron y Zaninetti, 1972a y Palaxius con la Familia Thalassinidae. Actualmente Palaxius es producido por el género $\mathrm{Ca}$ llianassa (Blau y Grün, 2000), conociéndose también esta asociación en el registro fósil (Peckmann et al., 2007).

Teniendo en cuenta la distribución de los crustáceos fósiles en el Grupo Mendoza, la arquitectura de 


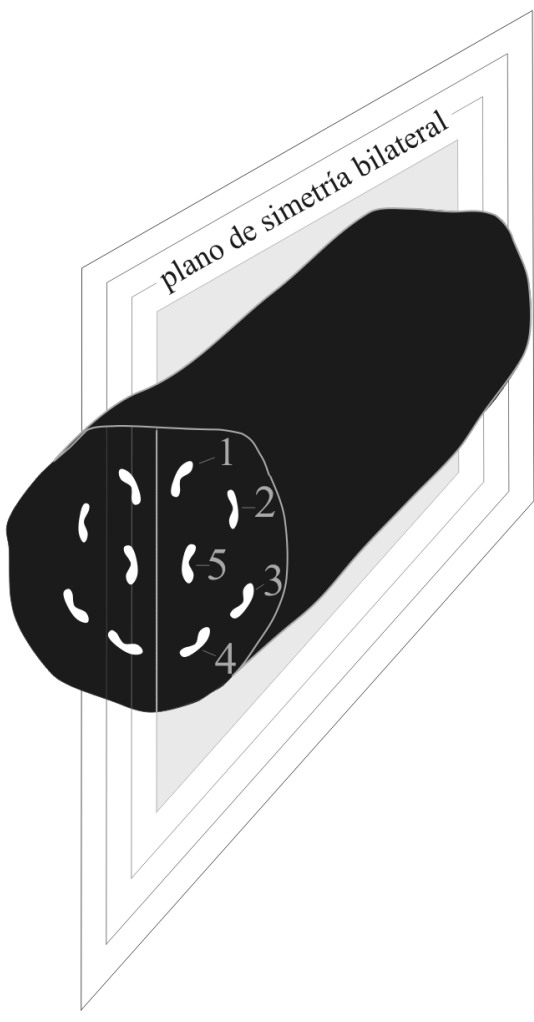

Figura 3. Diagrama esquemático de la icnoespecie Palaxius decaochetarius Palik / schematic diagram of Palaxius decaochetarius Palik.

las galerías de Thalassinoides y los datos bibliográficos que relacionan la morfología de los microcoprolitos con sus posibles productores (e.g. Blau y Grün, 2000; Peckmann et al., 2007), puede inferirse que los microcoprolitos del icnogénero Palaxius en la Formación Vaca Muerta fueron producidos por callianássidos.

\section{Ambiente sedimentario}

Actualmente se tiene registro de microcoprolitos de crustáceos en casi todos los ambientes de aguas tranquilas. Durante el Jurásico y Cretácico aparecen principalmente asociados a sedimentos blandos de ambientes marinos someros (Buchs et al., 2009). Sin embargo, algunos trabajos reportan la presencia de estos microcoprolitos en ambientes de rampa externa del Jurásico Inferior y Cretácico Superior (Schweigert et al., 1997; De Romero y Galea-Álvarez, 1995). Más recientemente se reconoció la presencia de Palaxius y Favreina en depósitos del Jurásico Superior (Oxfordiano) y Paleoceno-Eoceno asociados a sistemas hidrotermales de fondo oceánico (Peckmann et al., 2007; Senowbari-Daryan et al., 2007; Buchs et al., 2009).

En la Formación Vaca Muerta la distribución de los sistemas de Thalassinoides está asociada principal- mente a las facies de rampa media distal y rampa externa proximal (Kietzmann et al., 2008; Kietzmann y Palma, 2009) en tanto que la presencia de microcoprolitos suele estar asociada a los depósitos de rampa externa.

Es importante destacar que en general los niveles con microcoprolitos no se encuentran asociados a otras trazas generadas por crustáceos, como por ejemplo Thalassinoides, cuyas galerías suelen estar exhumadas indicando procesos erosivos asociados a corrientes de fondo o eventos de tormenta (Kietzmann y Palma, 2008). Estas características indican la para-autoctonía de los microcoprolitos, probablemente producidos en sectores de rampa media distal-rampa externa proximal, y luego transportados y acumulados en posiciones menos oxigenadas de la rampa externa.

Tradicionalmente se ha considerado que el ambiente sedimentario de la Formación Vaca Muerta estuvo sometido a constantes condiciones euxínicas (e.g. Legarreta y Uliana, 1991). Sin embargo, las extensas galerías de Thalassinoides presentes en determinados niveles de la unidad, así como el abundante contenido de microcoprolitos, ponen en duda esta tradicional concepción e indican que el grado de oxigenación tuvo variaciones a lo largo de los distintos sectores de la rampa.

\section{Conclusiones}

En este trabajo se presenta el primer registro de microcoprolitos de crustáceos para la Cuenca Neuquina y Argentina. Se describe la icnoespecie Palaxius decaochetarius Palik, que constituye el primer registro del icnogénero Palaxius para el Jurásico Superior y Cretácico Inferior de América del Sur.

Considerando la distribución de los crustáceos fósiles en el Grupo Mendoza, se infiere que el icnogénero Palaxius fue producido por callianásidos, siendo consistente con los datos de la literatura. Asimismo, la falta de asociación entre los mirocoprolitos y otras trazas generadas por crustáceos indica la para-autoctonía de los mismos, así como la presencia de sectores más oxigenados en la rampa carbonática.

\section{Agradecimientos}

Los autores agradecen especialmente a J. Blau por sus observaciones, a G. Schweigert, B. Senowbari-Daryan y D. Buchs por proporcionar material bibliográfico. A A. Concheyro por facilitar los microscopios con cámara fotográfica. A la editora C. Laprida y a los revisores N. Carmona y P. Pazos por su contribución al mejoramiento de esta nota. Este estudio fue financiado por los proyectos UBACyT X-133 y PIP 5142 (CONICET). 


\section{Bibliografía}

Aguirre-Urreta, M.B. 1989. The Cretaceous Decapod Crustacea of Argentina and the Antartic Peninsula. Palaeontology 32: 199-252.

Aguirre-Urreta, M.B. 2003. Early Cretaceous decapod Crustacea from the Neuquén Basin, west-central Argentina. Contributions to Zoology 72: 79- 81.

Bertling, M., Braddy, S.J., Bromley, R.G., Demathieu, G.R., Genise, J., Mikuláš, R., Nielsen, J.K., Nielsen, K.S.S., Rindsberg, A.K., Schlirf, M. y Uchman, A. 2006. Names for trace fossils: a uniform approach. Lethaia 39: 265-286.

Blau, J. y Grün, B. 2000. Liste der bekannten Gattungen und Arten. FAVRIS - interactive database on structured crustacean coprolites. http://www.uni-giessen.de gg13/FAVRIS/FAVRIS.htm

Blau, J., Grün, B. y Senff, M. 1993. Crustaceen-Koprolithen aus der Trias der westlichen Tethys (Lienzer Dolomiten, Österreich; Pragser Dolomiten, Italien) und vom Gondwana-Westrad (oberes Magdalenatal, Kolumbien, Südamerika). Paläontologische Zeitschrift 67: 193-214.

Blau, J., Moreno, M. y Senff, M. 1995. Palaxius caucaensis n. sp., a crustacean microcoprolite from the basal Nogales Formation (Campanian to Maastrichtian) of Colombia. Micropaleontology 41: 85-88.

Blau, J., Rosas, S. y Senff, M. 1994. Favreina peruviensis n. sp., ein Crustaceen-Mikrokoprolith aus dem Lias von Peru. Paläontologische Zeitschrift 68: 521-527.

Bromley, R.G. 1990. Trace Fossils. Biology and Taphonomy. Unwin Hyman, London, $280 \mathrm{pp}$.

Brönnimann, P. 1955. Microfossils incertae sedis from the Upper Jurassic and Lower Cretaceous of Cuba. Micropaleontology 1: 28-51.

Brönnimann, P. y Norton, P. 1960. On the classification of fossil fecal pellets and description of new forms from Cuba, Guatemala and Libya. Eclogae Geologicae Helvetiae 53: 832-842.

Brönnimann, P., Caron, J.P. y Zaninetti, L. 1972a. New galatheid anomuran (Crustacea, Decapoda) coprolites from the Rhetian of Provence, southern France. Mitteilungen der Gesellschaft der Geologie- und Bergbaustudenten in Wien 21: 905-920.

Brönnimann, P., Caron, J.P. y Zaninetti, L. 1972b. Parafavreina, n.gen. n.sp., a new thalassinid anomuran (Crustacea, Decapoda) coprolite form-genus from the Triassic and Liassic of Europa and North Africa. Mitteilungen der Gesellschaft der Geologie- und Bergbaustudenten in Wien 21: 941-956.

Buchs, D.M., Guex, J., Stucki, J. y Baumgartner, P.O. 2009. Paleocene Thalassinidea colonization in deep-sea environment and the coprolite Palaxius osaensis n. ichnosp. in Southern Costa Rica. Revue de micropaléontologie 52: 123-129.

De Romero, L. y Galea-Álvarez, F.A. 1995. Campanian Bolivinoides and microfacies from the La Luna Formation, Venezuela. Marine Micropaleontology 26: 385-404.

Ehrenberg, K. 1944. Ergänzende Bemerkungen zu den seinerzeit aus dem Miozän von Burgschleinitz beschriebenen Gangkernen und Bauten dekapoder Krebse. Paläontologische Zeitschrift 23: 354-359.

Förster, R. y Hillebrandt, A.V. 1984. Das Kimmeridge des Profeta-Jura in Nordchile mit einer Mecochirus-Favreina-Vergesellschaftung (Crustacea, Decapoda Ichnogenus). Mitteilungen der Bayeren Staatssammulgen in Paläontologie und Geologie 24: 67-84.

Kietzmann, D.A. y Palma, R.M. 2009. Tafofacies y biofacies de Formación Vaca Muerta en el sector surmendocino de la Cuenca Neuquina: implicancias paleoecológicas, sedimentológicas y estratigráficas. Ameghiniana 46: 321-343.

Kietzmann, D.A., Palma, R.M. y Bressan, G.S. 2008. Facies y microfacies de la rampa tithoniana-berriasiana de la Cuenca Neuquina (Formación Vaca Muerta) en la sección del Arroyo LoncocheMalargüe, provincia de Mendoza. Revista de la Asociación Geológica Argentina 63: 696-713.

Kristan-Tollmann, E. 1989. Agantaxia biserialis n.g. sp., ein Anomuren-Koprolith aus dem tithonen Plattenkalk von Ober-Österreich. Neues Jahrbuch für Geologie und Paläontologie 1989: 23-29.

Leanza, H.A. 1973. Estudio sobre los cambios faciales de los estratos limítrofes Jurásico-Cretácicos entre Loncopué y Picun Leufú,
Provincia del Neuquén, República Argentina. Revista de la Asociación Geológica Argentina 28: 97-132.

Leanza, H. y Zeiss, A., 1990. Upper Jurassic Lithographic Limestones from Argentina (Neuquén Basin): Stratigraphy and Fossils. Facies 22: $169-186$

Legarreta, L. y Kozlowski, E. 1981. Estratigrafía y sedimentología de la Formación Chachao, Provincia de Mendoza. $8^{\circ}$ Congreso Geológico Argentino (San Luis), Actas 2: 521-543.

Legarreta, L. y Uliana, M.A. 1991. Jurassic-Cretaceous marine oscillations and geometry of back-arc basin fill, central Argentine Andes. Internacional Association of Sedimentologist, Special Publication 12, London, pp. 429-450.

Mombrú, C.A., Uliana, M.A. y Bercowski, F. 1978. Estratigrafía y sedimentología de las acumulaciones biocarbonáticas del Cretácico Inferior surmendocino. $7^{\circ}$ Congreso Geológico Argentino (Neuquén), Actas 1: 685-700.

Moore, H.B. 1939. Faecal pellets in relation to marine deposits. En: P. D. Trask (ed.), Recent marine sediments. A symposium, p. 49-65. London.

Palik, P. 1965. Remains of crustacean excrement from the lower Cretaceous of Hungary. Micropaleontology 11: 98-104.

Palma, R.M. y Lanés, S. 2001. Shell Bed Stacking Patterns in the Chachao Formation (early Valanginian) in Malargüe Area, Mendoza Province, Neuquén Basin-Argentina. Carbonates and Evaporites 16: 168-180.

Palma, R.M., Bressan, G.S. y Kietzmann, D.A. 2008. Diagenesis of bioclastic oyster deposits from the Lower Cretaceous (Chachao Formation), Neuquén Basin, Mendoza Province. Carbonates and Evaporites 23: 39-49.

Peckmann, J., Senowbari-Daryan, B., Birgel, D. y Goedert, J.L. 2007. The crustacean ichnofossil Palaxius associated with callianassid body fossils in an Eocene methane-seep limestone, Humptulips Formation, Olympic Peninsula, Washington. Lethaia 40: 273-280.

Riccardi, A.C., Leanza, H.A., Damborenea, S., Manceñido, M., Ballent, S. y Zeiss, A. 2000. Marine Mesozoic Biostratigraphy of the Neuquén Basin. En: H. Miller y F. Hervé (eds.), Zeitschrift fur Angewandte Geologie, Hannover, $31^{\circ}$ Internacional Geological Congress, SH 1: 103-108.

Scasso, R.A, Alonso, S.M, Lanés, S, Villar, H.J. y Lippai, H. 2002. Petrología y geoquímica de una ritmita marga-caliza del Hemisferio Austral: El Miembro Los Catutos (Formación Vaca Muerta), Tithoniano medio de la Cuenca Neuquina. Revista de la Asociación Geológica Argentina 57: 143-159.

Schweigert, G., Seegis, D.B., Fels, A. y Leinfelder, R.R. 1997. New internally structured decapod microcoprolites from Germany (Upper Triassic/Lower Miocene), southern Spain (Lower/Middle Jurassic) and Portugal (Upper Jurassic); taxonomy, palaeoecology and evolutionary implications. Paläontologische Zeitschrift 71: 51-69.

Senowbari-Daryan, B. y Kube, B. 2003. The ichnogenus Palaxius (crustacean coprolite) and description of $P$. hydranensis $\mathrm{n}$. $\mathrm{sp}$. from the Upper Triassic (Norian part of 'Pantokrator'-limestone) of Hydra/Greece. Paläontologische Zeitschrift 77: 115-122.

Senowari-Daryan, B. y Kuss, J. 1992. Anomuren-Koprolithen aus der Kreide von Ägypten. Mitteilungen des Geologisch-Paläontologischen Instituts der Universität Hamburg 73: 129-157.

Senowbari-Daryan, B. y Stanley, G.D., 1986. Thalassinid anomuran microcoprolites from Upper Triassic carbonate rocks of Central Peru. Lethaia 19: 343-354.

Senowbari-Daryan, B., Gaillard, C. y Peckmann, J. 2007. Crustacean microcoprolites from Jurassic (Oxfordian) hydrocarbon-seep deposits of Beauvoisin, southeastern France. Facies 53: 229-238.

Vialov, O.S. 1978. Favreinidae (coprolites of Crustacea) from Turonian of the Lower Amudaria. Paleontologiceskij sbornik 15: 58-67.

Weaver, C. 1931. Paleontology of the Jurassic and Cretaceous of West Central Argentine. Memoir University of Washington 1, 469 p., Seattle.

Recibido: 23 de junio de 2009.

Aceptado: 19 de septiembre de 2009. 